\title{
Determining Confusion for Traditional and Experimental Pedestrian Signals in Rural and Suburban Areas in the United States
}

\author{
Wakeel Idewu ${ }^{1}$, Dogucan Mazicioglu ${ }^{1} \&$ Hana Naghawi ${ }^{2}$ \\ ${ }^{1}$ Department of Civil and Environmental Engineering, Virginia Military Institute, Virginia, USA \\ ${ }^{2}$ Civil Engineering Department, School of Engineering, The University of Jordan, Amman, Jordan \\ Correspondence: Hana Naghawi, Civil Engineering Department, School of Engineering, The University of Jordan, \\ Amman 11942, Jordan. Tel: 962-6535-5000. E-mail: h.naghawi@ju.edu.jo
}

Received: July 27, 2018

Accepted: August 8, 2018

Online Published: August 14, 2018

doi:10.5539/mas.v12n9p87

URL: https://doi.org/10.5539/mas.v12n9p87

\begin{abstract}
Walking is a mode of travel used by billions of people daily. Facilities that promote walking such as crosswalks, often involve sharing space with conflicting vehicular traffic. These areas are not immune to receiving pedestrians that either do not obey or do not understand today's pedestrian signals, which are used to communicate periods of safe crossing. Therefore, improving comprehension would subsequently improve safety and crash rates. The Traditional Pedestrian Signal in the United States displays an illuminated man and a hand to indicate a cautionarycrossing period, a transition period, and a crossing-prohibited period. This signal type was evaluated and compared to a relatively new Experimental Pedestrian Signal. The Experimental Pedestrian Signal presented in this paper utilized the figure of a walking man changing only by the colors green, yellow, and red. Both signals were analyzed to identify the phases that best communicated the intended action. Video files depicting a Traditional and Experimental Signal were administered with a questionnaire to test the comprehension of rural and suburban participants. The results indicated that the Experimental Pedestrian Signal was not better understood than the current Traditional Signal, although a vast majority of participants preferred the Experimental Signal. The lowest comprehension occurred during the transition phase for both pedestrian signal types. The results also suggest that the interpretation of the yellow color varies by location and may invoke mixed responses if incorporated in pedestrian signals. A more appropriate solution may be to combine both symbolic and color cues into future pedestrian signals.
\end{abstract}

Keywords: pedestrian signals, pelican signals, pedestrian safety, intersection crossing

\section{Introduction}

Walkable connected neighborhoods and communities continue to be at the forefront of transportation and government officials' plans. Today's environmental and economic climate has promoted enhancing common modes of travel such as walking. Properly functioning instrumentation, like pedestrian signals, are paramount to the day-to-day pedestrian travel - especially in areas with high vehicular activity. Pedestrian related fatalities occur at higher rates in areas without crosswalks (Shankar \& Umesh, 2003) (Chang, 2008), signifying the need for effective crosswalks and their accompanying pedestrian signals. Unfortunately, signals have been found to be insufficient with high rates of non-compliancy and reported injuries near crosswalks (Hatfield et al., 2007). Past studies have shown that pedestrians often resist waiting for their right-of-way to be honored at an intersection (Kruszyna et al., 2006). Others note that pedestrians sometimes decide to cross midblock because of the perceived inconvenience associated with crossing at the intersection (Dunlap and Associates, Incorporated, 2007).

In recent years, research addressing pedestrian confusion has been conducted using countdown signals. Visible countdown timers on a pedestrian signal box inform pedestrians of the time remaining before vehicular traffic commences. Prior to the inclusion of countdown timers, the signals mostly operated with the use of simple regulatory symbols. The three primary symbols used in communicating "walk" to "don't-walk" periods were:

1. A walking person

2. A flashing hand

3. A steady hand

Presently, the same phases exist, but countdown timers are now included during the Flashing Don't Walk period 
(FDW). Past research has shown that countdowns improve compliance rates and consequently safety (Kruszyna et al., 2006). The Manual on Uniform Traffic Control Devices (MUTCD) now requires pedestrian signals with countdowns be installed in crosswalks that require seven seconds or more to cross (Chang, 2008). However, there still remains a high crash risk where pedestrians and vehicles interact near intersection in the United States and abroad.

(Schroeder et al., 2009) and (Virkler, 1998) studied the behavior of pedestrians at crosswalks near intersections and midblock. They found high noncompliance rates and attributed it to many factors, namely the impatience of pedestrians desiring to cross. It was also found that along with age, sex, group size, and pedestrian flow, the symbol shown on pedestrian signals was also significant in the compliance rate (Brosseau et al., 2013). Because of this, evaluating the effectiveness of pedestrian signal phasing requires more investigation and a multifaceted solution to decreasing pedestrian injuries.

In 2012, approximately 80,743 pedestrians were either killed or injured in a traffic crash (NHTSA's National Center for Statistics and Analysis, 2014). This equated to a traffic related pedestrian injury every 7 minutes in the U.S. While over three-fourths of pedestrian fatalities occur at non-intersection locations, those pedestrian fatalities that do occur at intersections happen within the crosswalk at a high rate. This indicates communication at those intersections may be ignored or poorly understood by some. The research in this paper focuses on the latter to determine how well the displayed pedestrian signal messages are understood by potential users.

\subsection{The Color-Coded Pedestrian System}

The phases in a Traditional Pedestrian Signal used in the United States are Walk (W), Flashing Don't Walk (FDW) and Don't Walk (DW). Practices in some other countries focus more on color changes as opposed to the symbolic changes that are used in the U.S. when activated by a push button, a figure of a walking man (colored green) appears on the pedestrian signal, indicating it is safe to cross. When time of safe crossing expires, a figure of a standing man, colored red, replaces the previous figure. Countries that use color-coded pedestrian crossing systems include: New Zealand, Panama, Mexico, Peru, China and more. These signals match the green, red color system that is commonly used for controlling vehicular traffic. This type of signal is rarely observed in the United States and will be referred to as Experimental Pedestrian Signal. Both pedestrian signal types are shown below in Figure 1, and both were tested for comprehension in this study.

Although color-coded pedestrian signals seem to operate effectively, strategies successfully practiced in neighboring and distant countries are not easily transferable to the United States. Continued evidence of unsafe crossing practices in the U.S., warrants the need to enhance crosswalk features. Although more frequently implemented elsewhere, adopting a color-coded strategy in the U.S. may be beneficial since vehicular traffic responds favorably to the color-coded signal method. Familiarity with the red, yellow, green traffic signal convention remains a widely understood traffic pattern throughout the many years of road design advancements in U.S. This resulted in safer roadway networks as time progressed. However, of all transportation fatalities, 40 50 percent of them in big cities are pedestrian related (Van Houten et al., 2013). Moreover, some Traditional Pedestrian Signal features are still not intuitive and understood by many users. For instance, the top half of Figure 1 shows a Traditional Pedestrian Signal in the United States. Shown are three stages. The middle stage would show a flashing hand and a countdown timer - signaling don't walk. This Flashing Don't Walk (FDW) stage is the most troublesome to understand, and is the cause for low compliance rates in a couple of studies. Xiong et al. (2014) found that low percentage of pedestrians understood the flashing signal phase, also (DeLaere et al., 2015) noted the insignificance FDW signals had on understanding the appropriate time to cross.

Adding countdown signals have increased compliance and safety at crosswalks with pedestrian signals. Despite the benefits, vision impaired pedestrians face a particular challenge when using these signals. Scott et al. (2012) found that people with vision acuity issues successfully identify phase changes with 87 percent accuracy, but they have a significantly more difficult time recognizing the countdown display.

Color variations in pedestrian signals can provide an additional line of communication and improve management of safe and unsafe crossing periods. That's not to say that all color coded pedestrian signals are sufficient. Just the simple presence of vehicles and pedestrians in the same space, regardless of safety countermeasures, can still result in crashes with pedestrians. This was the case in the study performed by (Quistberg, et al., 2014) where colorcoded pedestrian signals in Peru were not sufficient by themselves in decreasing crashes. In fact, this study and others like it (Brosseau et al., 2013) identified signal cycle lengths and pedestrian waiting times as major factors in risky pedestrian maneuvers leading to crashes. 


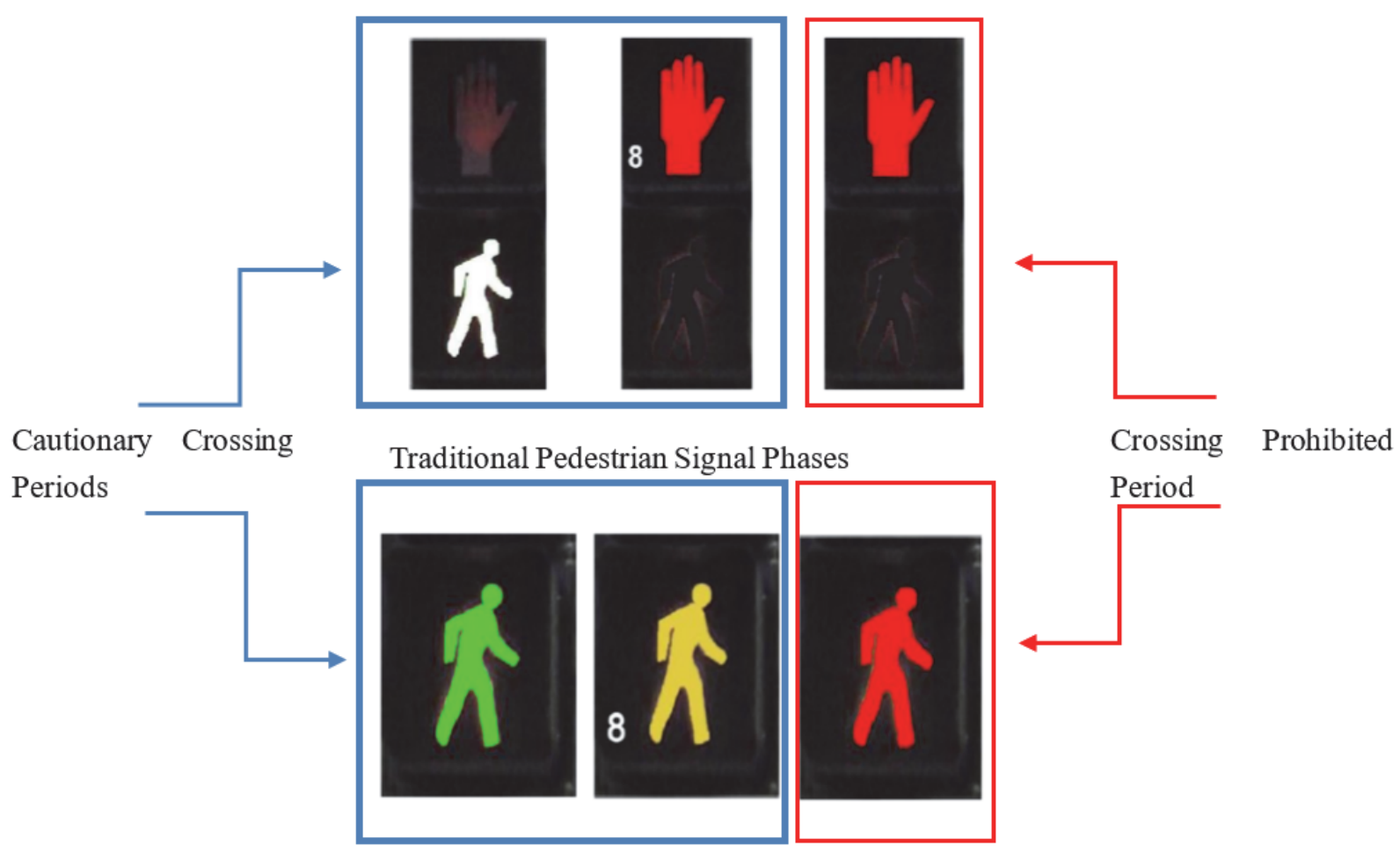

Experimental Pedestrian Signal Phases

Figure 1. Traditional and Experimental Pedestrian Signal Phases

\section{Pedestrian Signal Experiment}

This paper introduced a "new" pedestrian signal to a group of participants in the U.S. and compared their ability to select the appropriate response to the signals shown (in stages). Both the Traditional and Experimental Pedestrian Signals were shown to participants whose questionnaire responses were later compared and evaluated.

Unlike the Traditional Pedestrian Signal that changes from the figure of a man to a flashing, then steady hand, the Experimental Pedestrian Signal used a figure of a man, changing only by color (red, yellow, or green). The figures shown in Figure 1 were superimposed onto video images that were used in conjunction with a paper-based questionnaire. A countdown timer was used in the videos and showed the number of seconds remaining (beginning at 10 seconds) during the transition between the "walk" phase and "do not walk" phase. The phase shown in the middle of Figure 1 represents the countdown timer showing eight seconds left. The phases shown in the top half of Figure 1 are in accordance with rules outlined in the Manual on Uniform Traffic Control Devices (MUTCD, 2009), and it, along with the Experimental Signal, was clearly shown on video, in progressive order, to the survey participants. The surveying methods used in comparing the two pedestrian signal plans are explained in the sections that follow.

\section{Survey}

A video file and an accompanying questionnaire were created and administered to fourth graders in rural and suburban areas. Fourth graders were selected as participants for this study because they were thought to possess the capacity to comprehend questions displayed in the survey while lacking substantial exposure to pedestrian crosswalks with signals. Using this group decreased the chance of pre exposure and biased opinion skewing the responses. This approach would allow researchers to compare the effectiveness of both pedestrian signal types at conveying the intended meaning.

A total of 178 participants were surveyed, of which 96 were from a suburban area and 82 were from a rural area. A video file, used in combination with a hardcopy questionnaire, was created at the pedestrian crosswalk located at the intersection of Jamestown Road and John Tyler Lane, Williamsburg, Virginia. The questionnaire included a total of twelve questions- three of them were basic demographic related. Questions 4 through 11 were divided into two parts. Part one, questions 4a through 11a, tested the understandability of the Traditional and the Experimental Pedestrian Signals. Part two, questions $4 \mathrm{~b}$ though $11 \mathrm{~b}$, were used to create the confusion scale in which the 
participants were asked to report how sure they were of their answers. The last question was an open-ended question in which the participants were asked to select their preferred pedestrian signal and to write a brief description explaining the reason for their selection.

The video file consisted of two different video scenarios, randomly ordered per group showing. Each group viewed both scenarios. One scenario presented the Traditional Signal phases and the other showed the Experimental Signal phases. The Traditional and Experimental Pedestrian Signals were superimposed onto the videos of each scenario and played for the survey participants. A snapshot of one video scenario is shown in Figure 2. In both scenarios, an individual is shown approaching a crosswalk (Figure 2a). The individual presses the push button located on the pedestrian signal pole then waits for the signal to change from a crossing-prohibited period (Figure 2d) to a crossing period (Figure $2 \mathrm{~b}$ ). The signal is later shown transitioning (Figure 2c) back to a crossing prohibited period.

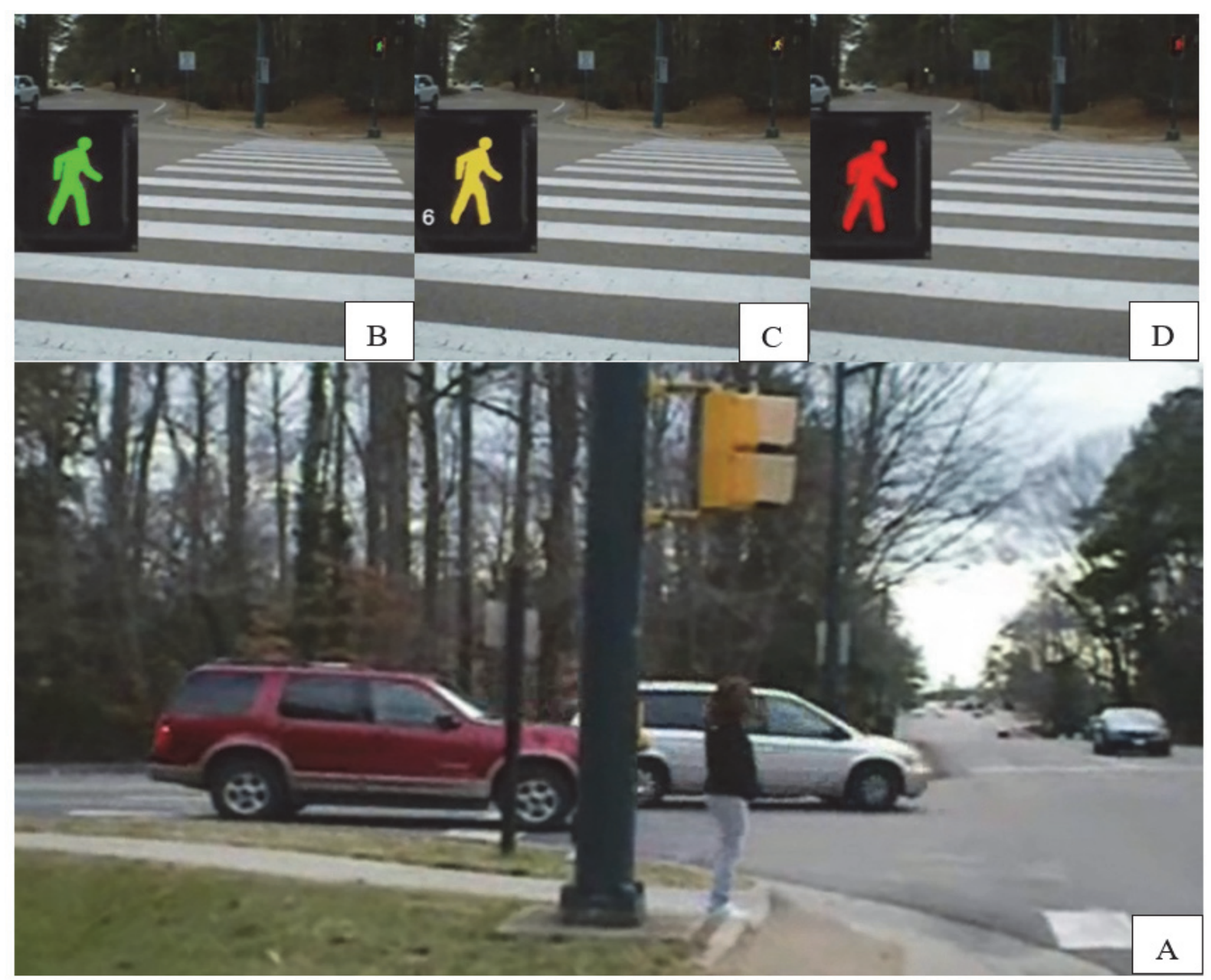

Figure 2. Snapshots of the video file image showing the approach to the crosswalk (a) Crossing Permitted Phase (b) Cautionary Crossing Phase (c) Crossing Prohibited Phase (d)

There were three possible ways to respond to the questions presented in the questionnaire: "Cross the Street", "Cross the Street Carefully", "Don't Cross the Street", but there was only one right answer in accordance with the MUTCD. Answers considered to be reasonably correct were also included in the analysis. An example of the question structure is shown in Figure 3 and the correct answers to all questions, supported by the rules and regulations in the MUTCD, are displayed in Figure 4. The correct answers noted in the MUTCD are conditional, and are only considered correct if the pedestrian has not yet entered the pedestrian crosswalk before viewing the displayed symbol. In the instance a pedestrian notices the signal after beginning to cross, he/she should continue crossing the intersection if they started crossing prior to the blinking/flashing hand phase (MUTCD, 2009). 
4. a) What would you do if you saw this signal

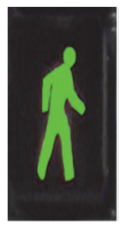

b) How sure are you of your answer above
○ Very Sure
- Somewhat Sure
○ Not Sure At All

\section{5. a) what would you do if you saw this signal}

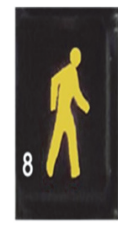

- Don't cross the street

- Cross the street

- Cross the street carefully
- Very Sure
- Somewhat Sure
- Not Sure At All

Figure 3. Example of Survey Questionnaire Question

\begin{tabular}{|c|c|c|c|c|c|c|c|c|}
\hline Question & $4 a$ & $5 a$ & $6 a$ & $7 a$ & $8 \mathrm{a}$ & $9 a$ & $10 \mathrm{a}$ & $11 \mathrm{a}$ \\
\hline & Lit Man & $\begin{array}{l}\text { Flashing } \\
\text { Hand } 8 \\
\text { seconds } \\
\text { remaining }\end{array}$ & $\begin{array}{l}\text { Flashing } \\
\text { Hand } 1 \\
\text { seconds } \\
\text { remaining }\end{array}$ & $\begin{array}{l}\text { Steady } \\
\text { Red } \\
\text { Hand }\end{array}$ & $\begin{array}{l}\text { Lit Green } \\
\text { Man }\end{array}$ & $\begin{array}{l}\text { Yellow } \\
\text { Flashing } \\
\text { Man } 8 \\
\text { seconds } \\
\text { remaining }\end{array}$ & $\begin{array}{l}\text { Yellow } \\
\text { Flashing } \\
\text { Man } 1 \\
\text { second } \\
\text { remaining }\end{array}$ & $\begin{array}{l}\text { Lit Red } \\
\text { Man }\end{array}$ \\
\hline $\begin{array}{l}\text { Symbol } \\
\text { Shown on } \\
\text { Survey }\end{array}$ & & & & & & & & \\
\hline $\begin{array}{l}\text { Correct } \\
\text { (MUTCD) } \\
\text { Answers }\end{array}$ & $\begin{array}{l}\text { Cross } \\
\text { Carefully }\end{array}$ & $\begin{array}{l}\text { Do Not } \\
\text { Cross }\end{array}$ & $\begin{array}{l}\text { Do Not } \\
\text { Cross }\end{array}$ & $\begin{array}{l}\text { Do Not } \\
\text { Cross }\end{array}$ & $\begin{array}{l}\text { Cross } \\
\text { Carefully }\end{array}$ & $\begin{array}{l}\text { Do Not } \\
\text { Cross }\end{array}$ & $\begin{array}{c}\text { Do Not } \\
\text { Cross }\end{array}$ & $\begin{array}{c}\text { Do Not } \\
\text { Cross }\end{array}$ \\
\hline $\begin{array}{l}\text { Reasonably } \\
\text { Correct } \\
\text { Answer }\end{array}$ & $\begin{array}{l}\text { Cross the } \\
\text { street } \\
\text { Or } \\
\text { Cross } \\
\text { Carefully }\end{array}$ & $\begin{array}{l}\text { Cross } \\
\text { Carefully } \\
\text { Or } \\
\text { Do Not } \\
\text { Cross } \\
\end{array}$ & $\begin{array}{l}\text { Do Not } \\
\text { Cross }\end{array}$ & $\begin{array}{l}\text { Do Not } \\
\text { Cross }\end{array}$ & $\begin{array}{l}\text { Cross the } \\
\text { street } \\
\text { Or } \\
\text { Cross } \\
\text { Carefully }\end{array}$ & $\begin{array}{l}\text { Cross } \\
\text { Carefully } \\
\text { Or } \\
\text { Do Not } \\
\text { Cross } \\
\end{array}$ & $\begin{array}{l}\text { Do Not } \\
\text { Cross }\end{array}$ & $\begin{array}{l}\text { Do Not } \\
\text { Cross }\end{array}$ \\
\hline
\end{tabular}

Figure 4. Correct Answers to Survey Questions

The survey video was played and paused by a moderator at different stages to ask participants questions about their response to a specific signal phase from questions 4 to 11. The participants were instructed to watch the video and answer the survey questions as if they observed the signal phases before entering the intersection. The answers obtained from the questionnaires provided information to identify and derive the following:

1. The Pedestrian Signal that was best understood overall and therefore better fitting for use near busy intersections.

2. The phases for Traditional and/or Experimental Signals that best explained the desired movements.

3. Limitations of the Experimental and Traditional Pedestrian Signal.

4. The elements of the pedestrian signals that were either not easily understood or not being obeyed.

5. The differences between the responses of suburban and rural participants.

6. Recommendations for further evaluation of the Experimental Signal and improving the currently used signal to increase comprehension.

\section{Survey Results}

Because participants' previous experience with pedestrian signals could bias results, general statistics related to the studied population and their exposure to pedestrian signals were gathered for analysis. The majority of the participants $(67 \%)$ responded to having seen a crosswalk with a pedestrian signal three or more times. Additionally, 17 percent were exposed once or twice, while 16 percent never observed a pedestrian crosswalk with a signal. As 
expected, suburban area participants claimed to have observed a pedestrian crosswalk with a signal more often, with a difference of 15.5 percent, than rural area participants.

\subsection{Statistical Significance}

Statistical analyses of the data were performed by comparing the two population proportions at 95 percent confidence level to evaluate understanding of the Traditional and Experimental Pedestrian Signals. The following null and alternative hypotheses were used for a two-tailed hypothesis test:

- $\mathrm{H}_{\mathrm{o}}$ : students equally understand both the Traditional and Experimental Pedestrian Signals design.

- $\mathrm{H}_{1}$ : students didn’t equally understand both the Traditional and Experimental Pedestrian Signals design.

The bold italicized numbers shaded in rightmost columns of Table 1 and Table 2 show that a significant difference existed between the two pedestrian signal designs in rural areas for half of the signal phases, which means that students equally understood almost half of the signal phases for both the Traditional and Experimental Pedestrian Signal design.

The greatest significant difference for both rural and suburban participants concerned the transition phase (flashing hand or yellow illuminated man). As for suburban areas, there were no significant differences for almost all signal phases. Generally, this is evidence that students equally understood the Experimental and Traditional Pedestrian Signals design.

Table 1. Proportion of Participants Who Accurately Interpreted the Signal Phases in Rural Area

\begin{tabular}{|c|c|c|c|c|}
\hline \multirow[b]{2}{*}{ Participant Response } & \multicolumn{2}{|c|}{ Signal Phase } & \multirow[t]{2}{*}{ Test-Statistics } & \multirow[t]{2}{*}{ P-value } \\
\hline & Traditional $\%$ & Proposed $\%$ & & \\
\hline \multicolumn{5}{|l|}{ Questions $4 \mathrm{a}$ and $8 \mathrm{a}$} \\
\hline MUTCD Correct Answer & 0.5490 & 0.2440 & -3.9911 & $6 E-05$ \\
\hline Wrong Answer & 0.4512 & 0.7560 & & \\
\hline Reasonably Correct Answer & 0.9510 & 1.0000 & 2.0248 & 0.0434 \\
\hline \multicolumn{5}{|l|}{ Questions 5a and 9a } \\
\hline MUTCD Correct Answer & 0.7680 & 0.1340 & -8.1600 & 0.0000 \\
\hline Wrong Answer & 0.2317 & 0.8659 & & \\
\hline Reasonably Correct Answer & 0.9390 & 0.9150 & -0.6997 & 0.5485 \\
\hline \multicolumn{5}{|l|}{ Questions 6a and 10a } \\
\hline MUTCD Correct Answer & 0.9020 & 0.7930 & -1.9552 & 0.0500 \\
\hline Wrong Answer & 0.0976 & 0.2073 & & \\
\hline Reasonably Correct Answer & 0.9020 & 0.7930 & -1.9552 & 0.0500 \\
\hline
\end{tabular}




\begin{tabular}{ccccc}
\hline Questions 7a and 11a & & & & \\
& & & & \\
& & & & \\
MUTCD Correct Answer & 0.9150 & 0.9512 & 0.9365 & 0.3472 \\
Wrong Answer & 0.0854 & 0.0488 & & \\
Reasonably Correct Answer & 0.9150 & 0.9510 & 0.9365 & 0.3472 \\
\hline
\end{tabular}

Table 2. Proportion of Participants Who Accurately Interpreted the Signal Phases in Suburban Area

\begin{tabular}{|c|c|c|c|c|}
\hline \multirow[b]{2}{*}{ Participant Correct Response } & \multicolumn{2}{|c|}{ Signal Phase } & \multirow[t]{2}{*}{ Test-Statistics } & \multirow[t]{2}{*}{ P-value } \\
\hline & Traditional \% & Proposed $\%$ & & \\
\hline \multicolumn{5}{|l|}{ Questions 4a and 8a } \\
\hline MUTCD Correct Answer & 0.4480 & 0.3250 & -1.9327 & 0.0536 \\
\hline Wrong Answer & 0.5520 & 0.6670 & & \\
\hline Reasonably Correct Answer & 1.0000 & 0.9990 & 0.3099 & 0.7566 \\
\hline \multicolumn{5}{|l|}{ Questions 5a and 9a } \\
\hline MUTCD Correct Answer & 0.5940 & 0.1880 & -5.7688 & 0.0000 \\
\hline Wrong Answer & 0.4060 & 0.8020 & & \\
\hline Reasonably Correct Answer & 0.9800 & 0.9580 & -0.8296 & 0.4065 \\
\hline \multicolumn{5}{|l|}{ Questions 6a and 10a } \\
\hline MUTCD Correct Answer & 0.8440 & 0.8380 & -0.2281 & 0.4091 \\
\hline Wrong Answer & 0.1660 & 0.1560 & & \\
\hline Reasonably Correct Answer & 0.8440 & 0.8380 & -0.2281 & 0.4091 \\
\hline \multicolumn{5}{|l|}{ Questions 7a and 11a } \\
\hline MUTCD Correct Answer & 0.9690 & 1.0000 & 1.7367 & 0.0819 \\
\hline Wrong Answer & 0.0310 & 0.0000 & & \\
\hline Reasonably Correct Answer & 0.9690 & 1.0000 & 1.7367 & 0.0819 \\
\hline
\end{tabular}


Despite its perceived ability to convey a clear meaning, the Experimental Signal's yellow cautionary crossing period produced the lowest measure of comprehension. Yellow was the color of choice to include in the transition period because it mimicked traffic signal coloring and the meaning was thought to be widely understood.

\section{Confusion Scale}

A confusion matrix was created from the responses using what was considered to be reasonably correct answers. For each signal phase question (part a), participants were asked to respond how confident they were of their answer (part b) "Very Sure," "Somewhat Sure," or "Not Sure At All." This helped identify the phases that were most difficult to comprehend. Confusion percentages were then calculated and used to compare confusion across locales (rural and suburban areas). This process is explained in more detail below.

Question parts "a" and "b" were analyzed simultaneously and used to create the confusion percentages for each question. An example of a confusion scale matrix is shown below in Table 3. In this example 12.43 percent of participants who answered the question were regarded as confused. Highlighted in red and yellow, and explained in detail below, are the numbers used to derive the confusion percentage of 12.43 percent. It is important to note that all confusion percentages were inversely proportional to percentages of participants who answered the corresponding questions correctly. This suggests that the method used to derive and apply the confusion scale was suitable for analysis.

Table 3. Confusion Scale for Questions 6a/6b

\begin{tabular}{lllll}
\hline & $\begin{array}{l}\text { Correct } \\
\text { (Don't Cross) }\end{array}$ & $\begin{array}{l}\text { Wrong } \\
\text { (Cross) }\end{array}$ & $\begin{array}{l}\text { Wrong } \\
\text { (Cross Carefully) }\end{array}$ & Sum \\
\hline Very Sure & 125 & $\mathbf{2}$ & $\mathbf{6}$ & 133 \\
Somewhat Sure & 27 & 3 & $\mathbf{1 2}$ & 42 \\
Not Sure At All & 2 & 0 & 0 & $\mathbf{2}$ \\
Sum & & & Confusion Percentage $=$ & 177 \\
\hline & & & $\mathbf{1 2 . 4 3 \%}$ \\
\hline
\end{tabular}

Participants that claimed to be "Very Sure" of their answer but provided a wrong answer were regarded as confused. Individuals who responded "Not Sure At All" were also classified as confused, regardless of their answer, since they were thought to be guessing an action for the given signal phase. Lastly, participants who stated they were "Somewhat Sure" were classified as confused if they selected a wrong answer. The most frequently selected wrong answer was used in establishing confusion for "Somewhat Sure" responses. For example, the "Somewhat Sure" row in Table 3, shows 27 participants who chose the correct answer and 15 (3 and 12) participants who chose the wrong answer (Cross / Cross Carefully). However, the 12 participants who selected the wrong answers (Cross Carefully) were used in calculating the confusion scale. The wrong answer with the larger sample size was selected to remain conservative in the calculations. This action was also taken since people who were "Somewhat Sure" were assumed to be contemplating over two answers, which corresponds to the thought that if they were pondering over three answers, they would be "Not Sure At All". Similarly, if the participants were considering only one answer, they would be "Very Sure" of their response.

The confusion scale in this example was calculated by adding the cells highlighted in yellow/bold and dividing that number by the total number of responses (cell highlighted in red) before multiplying it by 100 to get a percentage. The method described above was applied to all pedestrian signal comprehension questions to generate Figure 5. The Traditional Signal phases shown in Figure 5 have lower confusion percentages than the Experimental Signal phase in all periods except the first and last phase. The most confusing phase in both cases, but more so for the Experimental Signal, was the transition phase showing one second remaining. 


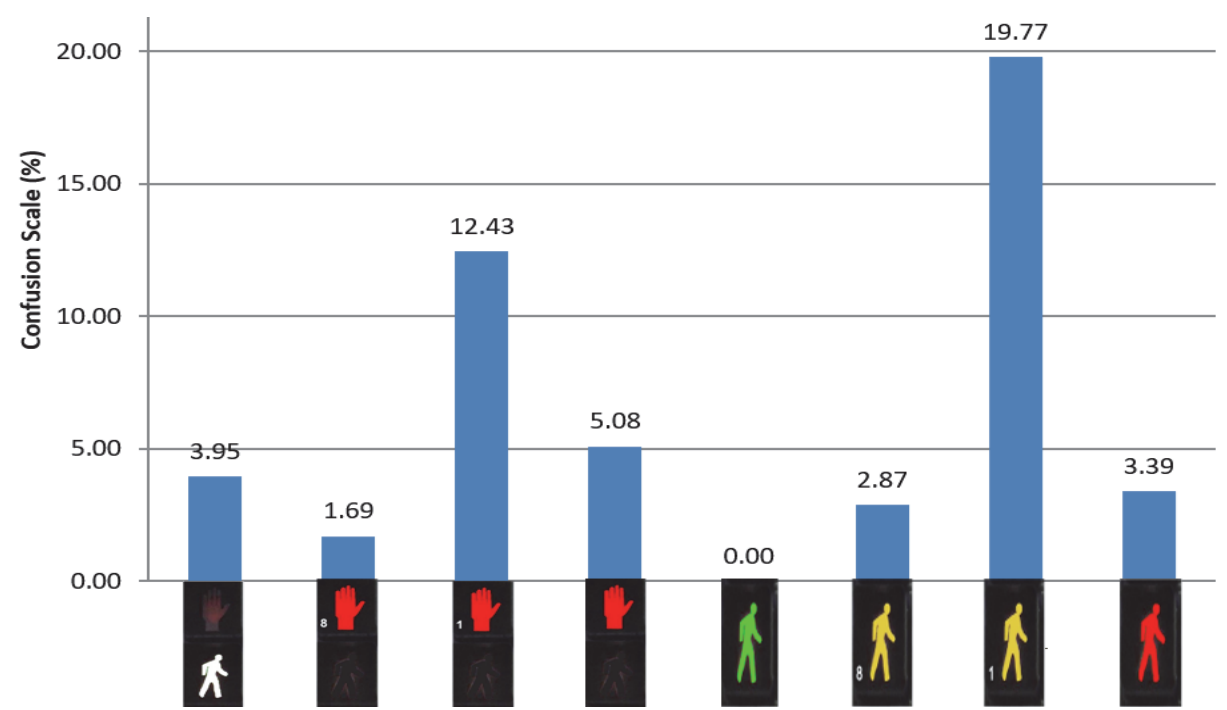

Figure 5. Confusion Percentages for Combined Rural and Suburban Participants

\subsection{Rural and Suburban Responses}

Results of the combined responses of rural and suburban area participants were presented in the previous sections. However, it was expected that participants from suburban areas would encounter and utilize pedestrian signals more frequently than participants from rural locations, and thus respond differently. As stated earlier 82 participants were from rural areas in or near Lexington, Virginia, while 96 participants resided in suburban areas in or near Richmond, Virginia. Figure 6 shows confusion comparisons of the two different area types, rural and suburban.

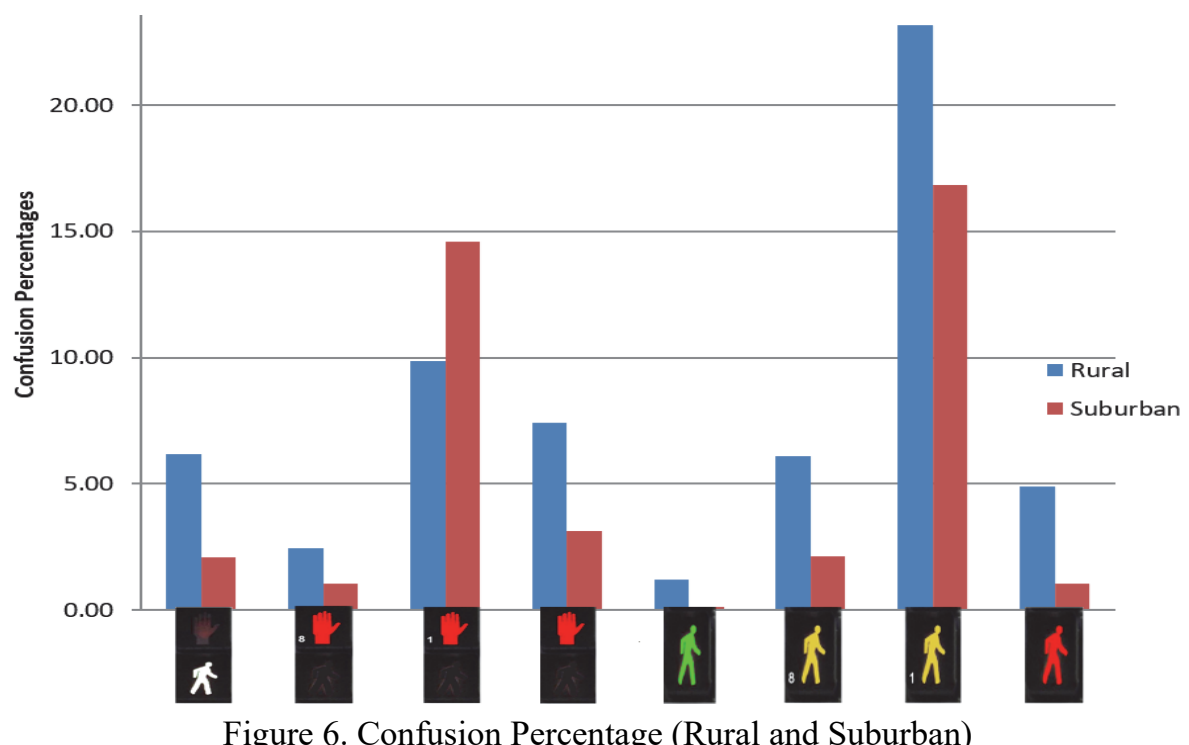

Due to their assumed increased exposure to the Traditional Pedestrian Signal, suburban area participants were expected to have lower confusion margins than the rural area participants. Figure 5 illustrates this was the case for all phases except the Traditional transition phase showing one second remaining.

The large disparity between both groups is for the question concerning a cautionary crossing period with eight seconds remaining (flashing red hand). The confusion level in this instance differs by 5 to 7 percentage points. This observation, along with the previous statistical comparisons mentioned earlier in this paper, suggests participants from suburban areas perceive the meaning of these particular phases differently than participants from rural areas.

Moreover, confusion scale percentages are relatively high for the prohibited crossing phases that require the most attention. The likelihood that these individuals with high levels of confusion would inappropriately react to the 
Traditional Signal could lead to dangerous encounters with approaching vehicles.

The last question of the survey instructed participants to select their preferred pedestrian signal and to write a brief description explaining the reason for their selection. A large percentage of the surveyed population, approximately 72 percent, preferred the Experimental Pedestrian Signal, despite the observed lack of understanding for the signal. The primary response for preferring the proposed Experimental Signal was its simplicity in conveying the necessary information by imitating the color coding displayed on modern traffic signals. Twenty eight percent of participants preferred the Traditional Signal, for its use of two symbols to communicate two allowed movements.

\section{Conclusion and Recommendations}

The current method used to guide pedestrians to and from intersection corners makes use of "Walk and Don't Walk" signals placed near the corners of intersections. These signals are designed to complement the vehicular traffic signals used to coordinate the travel movements of vehicles on the roadway. Pedestrian signals, although seemingly understandable, may be insufficient in communicating the allowed crossing periods. Although several features have been added to enhance pedestrian signalizing methods, there still remains incidents of pedestrians not conforming to the rules and allowed movements displayed on pedestrian signals. This non-compliancy has the potential to result in severe pedestrian-to- vehicle crashes and may be linked to one of two reasons:

1. Pedestrians are deliberately disobeying crossing rules and periods, or

2. Pedestrian fail to notice or do not fully understand the messages and meanings of symbols displayed on current pedestrian signals.

Some practices abroad involve color-coding pedestrian signals to reflect a more familiar traffic management approach. An Experimental Pedestrian Signal following this color coordination was virtually constructed and compared to a pedestrian signal traditionally used in the United States. Unlike the Traditional Pedestrian Signal that changes from a walking-man symbol to a flashing, then steady red hand, the Experimental Pedestrian Signal used a walking-man symbol, changing only by the colors green, yellow, and red. The objective was to identify the pedestrian signal and phases that were best understood by the surveyed population and that could lead to higher comprehension. A better understood pedestrian signal would decrease instances of pedestrians crossing intersections during unsafe and potentially harmful periods.

A video displayed phases of both the Traditional and Experimental Pedestrian Signals. It was shown to elementary school students who responded to what they interpreted seeing on a corresponding questionnaire. Specifically, fourth grade students were targeted as survey participants because their limited exposure to pedestrian signals decreased the chance of biasness, and because their age group was considered mature enough to complete the questionnaire.

The Experimental Pedestrian Signal was expected to be less confusing due to participants familiarity with the colors used in today's traffic lighting framework. However, the Experimental Signal was not better understood as initially hypothesized. The yellow phase for the Experimental Signal seemingly caused misunderstanding and confusion. Observations from the results suggest that the participants believed that the yellow color signified a phase of expedited crossing rather than the intended meaning of slowing down and stopping before entering the intersection.

As a whole, the signal phase that was least understood for both pedestrian signals was the cautionary-crossing phase, which is used to transition from crossing permitted to the crossing prohibited phase. The degree to which the transition phase (Flashing Don't Walk Hand or Yellow Lit Walking Man) was misunderstood depended on location. This suggests that rural and suburban participants interpreted the same transitional phase differently.

Despite evidence that confusion existed when the Experimental Signal was tested; the majority, approximately 72 percent, of the surveyed participants preferred the Experimental Signal over the Traditional Signal. The primary reason noted for this preference was the similarity between the color-coding schemes of the proposed signal and today's traffic signals, which participants thought provided a simpler and easier signal to understand. However, responses to the yellow transition phase suggest that the use of the color yellow would result in increased confusion due to varying interpretations of the yellow color's meaning. It is recommended that the color yellow not be used for future implementation of a color-coded pedestrian signal.

Instead, a combination of the Traditional and Experimental signal phase structure in suggested. The least confusing and the best-understood phase was the Experimental Signal's crossing permitted phase (green-lit man). The most confusing and least understood phase was the Experimental Signal's (trasition phase) yellow-lit walking man. Combining visually different symbols and specific conventional color-coding (green $=$ go, red $=$ stop) in pedestrian signals would enhance signal recognition, increase comprehension, and likely produce more favorable results. 


\section{Acknowledgments}

The authors of this paper acknowledge the support of the Colonial Heights Elementary School of Henrico County, Waddell Elementary School and Central Elementary of Rockbridge County.

\section{References}

Brosseau, M., Miranda-Moreno, L., Saunier, N., \& Zangenehpour, S. (2013). The Impact of Waiting Time and Other Factors on Dangerous Pedestrian Crossings and Violations at Signalized Intersections: A Case Study in Montreal. Transportation research part F: Traffic Psychology and Behaviour, 21, 159-172.

Chang, D. (2008). National Pedestrian Crash Report. U.S. Department of Transportation, U.S. Highway Safety Administration. Retrieved from https://crashstats.nhtsa.dot.gov/Api/Public/ViewPublication/810968

DeLaere, G., Van Houten, R., Morgan, J., \& Shurbutt, J. (2015). Countdown pedestrian signals and low-vision pedestrians. Transportation Research Record: Journal of the Transportation Research Board, 2492, 57-60.

Dunlap and Associates, Incorporated. (2007). A Compendium of NHTSA's Pedestrian and Bicyclist Traffic Safety Research Projects 1969-2007. NHTSA. Retrieved from https://www.ems.gov/pdf/HS810793.pdf

Hatfield, J., Fernandes, R., Job, R. F., \& Smith, K. (2007). Misunderstanding of right-of-way rules at various pedestrian crossing types: Observational study and survey. Accident Analysis and Prevention, 39, 833-842.

Kruszyna, M., Mackiewicz, P., \& Szydlo, A. (2006). Influence of Pedestrians' Entry Process on Pedestrian Delays at Signal-Controlled Crosswalks. Journal of Transportation Engineering, 855-861.

Manual on Uniform Traffic Control Devices. (2009, May 1). Retrieved from MUTCD. Retrieved from https://mutcd.fhwa.dot.gov/pdfs/2009r1r2/mutcd2009r1r2edition.pdf

NHTSA's National Center for Statistics and Analysis. (2014, April 1). Traffic Safety Facts: Pedestrians, April 2014. Retrieved from https://crashstats.nhtsa.dot.gov/Api/Public/ViewPublication/811888

Quistberg, D., Koepsell, T., Boyle, L., Miranda, J., Johnston, B., \& Ebel, B. (2014). Pedestrian Signalization and The Risk of Pedestrian-Motor Vehicle Collisions in Lima, Peru. Accident. Accident Analysis and Prevention, $70,273-281$.

Schroeder, B., Rouphail, N., \& Lehan, B. (2009). Observational Study of Pedestrian Behavior Along a Signalized Urban Corridor. Transportation Research Board 88th Annual Meeting. Washington, D.C.

Scott, A., Atkins, K., Bentzen, B., \& Barlow, J. (2012). "Perception of Pedestrian Traffic Signals by Pedestrians with Varying Levels of Vision.". Transportation Research Record, 57-64.

Shankar, \& Umesh. (April 2003). Pedestrian Roadway Fatalities. U.S. Department of Transportation.

Van Houten, R., Malenfant, L., Blomberg, R. D., Huitema, B. E., \& Casella, S. (2013). High-Visibility Enforcement on Driver Compliance With Pedestrian Right-of-Way Laws. (Report No. DOT HS 811 786). Washington, DC: National Highway Traffic Safety. Retrieved from https:/www.nhtsa.gov/sites/nhtsa.dot.gov/files/811786.pdf

Virkler, M. (1998). Pedestrian Compliance Effects on Signal Delay. Transportation Research Record 1636, 88-91.

Xiong, H., Xiong, L., Deng, X., \& Wang, W. (2014). Evaluation of the Impact of Pedestrian Countdown Signals on Crossing Behavior." (2014): 518295. Advances in Mechanical Engineering.

\section{Copyrights}

Copyright for this article is retained by the author(s), with first publication rights granted to the journal.

This is an open-access article distributed under the terms and conditions of the Creative Commons Attribution license (http://creativecommons.org/licenses/by/4.0/). 